\title{
Some Basic Characterization of the Function y
}

\author{
Rena Eldar Kizi Kerbalayeva ${ }^{1,2}$ \\ ${ }^{1}$ Institute of Mathematics and Mechanics, Azerbaijan National Academy of Sciences, Baku, Azerbaijan \\ ${ }^{2}$ School №227, Baku, Azerbaijan
}

Email address:

rena-kerbalayeva@mail.ru

\section{To cite this article:}

Rena Eldar Kizi Kerbalayeva. Some Basic Characterization of the Function $\gamma$, International Journal of Theoretical and Applied Mathematics. Vol. 7, No. 5, 2021, pp. 72-75. doi: 10.11648/j.ijtam.20210705.11

Received: August 28, 2021; Accepted: September 23, 2021; Published: October 28, 2021

\begin{abstract}
In this paper I have studied some characterization of the function $\gamma$. As in areas of Mathematics, we need a precise of given problem result in order to be absolutely clear. This paper seeks to do that and introduce new applications to aid our study. Some steps of the solutions to given paper in Basic Mathematics for the Analysis course involve arithmetic calculations that are too complicated to be performed mentally. In this paper I have included three Study Skills Checklists introduced to actively give how effectively use following views. The beginning of the paper has been introduced some properties of having sequences as a complete study this problem. In this instance, I have shown the actual computations that must be made to complete the formal prove. Hence than simply list the steps of arithmetic calculations making no mention of how the numerical values within the graphs are behaved, this unique feature will help answer often given question, from a interesting mathematics, "Is the function $\gamma$ rational?" Since information is often presented in the form of graphs, I need to be able to give some characterizations of a function of a natural-number argument (a sequence) and natural logarithmic (Napierian logarithms) function displayed in this way. It also serves as a method for the Euler transformations that I can perform immediately to solve the problem in this paper. Henceforth according to l'Hopital's rule one can easy to solve needing limit.
\end{abstract}

Keywords: A Limit, Functions of a Natural-number Argument, A Sequence, Transformations

\section{Introduction}

Constructive or computational methods have always been a characteristic feature of Analysis. The rapid development of new methods led a revival of the constructive methods, which are now used to investigate the mathematics structures. Is the function $\gamma$ rational? The problem is that answering the question we solve-how can we obtain this old problem from introducing? In the previous paper I studied about limit theory and I introduced the method of finding them. We have already known what a "limit" is. We knew a limit is a value, a function approaches, as the independent variable of the function gets nearer and nearer to a particular value. This paper also treats the sequence, the central concept of a function of a natural-number argument (a sequence). Here, I shall not be concerned with applications to Mathematics and Analysis, but instead will confine our study to general problem of the function $\gamma$. It also discuss briefly a sequence, a function of a natural-number argument (a sequence) and particular the Euler transformation, since these topics involve the solution of given problem. Here the sum of infinity series at a point of discontinuity is given by the arithmetic mean of the limit. Show by calculations of this limit of given infinity series that as the series is added together term by term the result approximates more and more closely to the remark of the function it represents $[1,3,5]$.

\section{Some Preliminaries}

Let us consider following math problem:

$$
\gamma=\lim _{n \rightarrow \infty}\left(\sum_{k=1}^{n} \frac{1}{k}-\ln n\right) .
$$

Here

$$
\left\{z_{n}\right\}=1+\frac{1}{2}+\frac{1}{3}+\cdots+\frac{1}{n}+\cdots-\ln n,
$$

and we hold 
Indeed,

$$
\sum_{k=1}^{n} \frac{1}{k}>\ln n, \text { for any } \mathrm{n} .
$$

$$
\sum_{k=1}^{n} \frac{1}{k}=\ln e^{\sum_{k=1}^{n} \frac{1}{k}}>\ln n
$$

due to

$$
e^{\sum_{k=1}^{n} \frac{1}{k}}>\ln n
$$

This sequence is numerical sequence. Henceforth we get

$$
\sum_{k=1}^{n} \frac{1}{k}-\ln n>0, \text { for any } \mathrm{n} .
$$

One can verify this sequence has limit, that is, this converges. [2, 4, 14]

Prove: We knew that,

$$
\frac{1}{n+1}<\ln \left(\frac{1+n}{n}\right)<\frac{1}{n}, \text { for any } \mathrm{n}
$$

We hold,

$$
\begin{gathered}
\frac{1}{2}<\ln 2<1, \text { if } \mathrm{n}=1, \\
\frac{1}{3}<\ln \frac{3}{2}<\frac{1}{2}, \text { if } \mathrm{n}=2, \\
\frac{1}{4}<\ln \frac{4}{3}<\frac{1}{3}, \text { if } \mathrm{n}=3, \\
\ldots \ldots \ldots \ldots \ldots \ldots \ldots \ldots \ldots \ldots \ldots \\
\frac{1}{n}<\ln \frac{n}{n-1}<\frac{1}{n-1}, \text { if any } \mathrm{n} .
\end{gathered}
$$

What shall we hold if we add first, second, third and n-th inequality? It is clear that, we shall get following inequality

$$
\begin{gathered}
\frac{1}{2}+\frac{1}{3}+\frac{1}{4}+\cdots+\frac{1}{n}< \\
\ln 2+\ln \frac{3}{2}+\ln \frac{4}{3}+\cdots+\ln \frac{n}{n-1}< \\
1+\frac{1}{2}+\frac{1}{3}+\cdots \frac{1}{n-1} .
\end{gathered}
$$

More precisely, according to following property $\ln a+$ $\ln b=\ln (a \times b)$, for any $\mathrm{a}>0, \mathrm{~b}>0$, then we have

$$
\frac{1}{2}+\frac{1}{3}+\frac{1}{4}+\cdots+\frac{1}{n}<\ln n<1+\frac{1}{2}+\frac{1}{3}+\frac{1}{4}+\cdots+\frac{1}{n-1} .
$$

From this inequality we hold

$$
\left\{\begin{array}{c}
\frac{1}{2}+\frac{1}{3}+\frac{1}{4}+\cdots+\frac{1}{n}-\ln n<0 \\
1+\frac{1}{2}+\frac{1}{3}+\frac{1}{4}+\cdots+\frac{1}{n-1}>\ln n
\end{array},\right.
$$

and consequently

$$
\left\{\begin{array}{c}
1+\frac{1}{2}+\frac{1}{3}+\frac{1}{4}+\cdots+\frac{1}{n}-\ln n<1 \\
1+\frac{1}{2}+\frac{1}{3}+\frac{1}{4}+\cdots+\frac{1}{n}-\ln n>\frac{1}{n}>0
\end{array} .\right.
$$

Then using inequality $\frac{1}{n+1}<\ln \left(\frac{1+n}{n}\right)<\frac{1}{n}$, we get

$$
0<\left\{z_{n}\right\}<1 \text {. }
$$

$$
z_{n+1}-z_{n}=1+\frac{1}{2}+\frac{1}{3}+\frac{1}{4}+\cdots+\frac{1}{n+1}-\ln (n+1)-
$$

$$
\begin{gathered}
\left(1+\frac{1}{2}+\frac{1}{3}+\frac{1}{4}+\cdots+\frac{1}{n}-\ln n\right)= \\
\frac{1}{n+1}-\ln (n+1)+\ln n= \\
\frac{1}{n+1}-\ln \left(\frac{n+1}{n}\right)<0 .
\end{gathered}
$$

It means, that $z_{n+1}<z_{n}$, more precisely given sequence decreases. We hold the sequence $\left\{z_{n}\right\}$ decreases and $z_{n}>0$, for any $\mathrm{n}$. It means that, $\lim _{n \rightarrow \infty}\left\{z_{n}\right\}=\mathrm{c}$ exits. In addition, we hold that $\mathrm{c}=\inf \left\{z_{n}\right\}, z_{n}>0, z_{n} \leq 1$ and $0<\mathrm{c}<1$. $[6,7,13]$.

\section{Main Results}

Here one cannot use some simple algebraic rules of limits

$$
\lim _{n \rightarrow \infty}\left(x_{n}-y_{n}\right)=\lim _{n \rightarrow \infty} x_{n}-\lim _{n \rightarrow \infty} y_{n} .
$$

Because of sequences $\left\{x_{n}\right\}$ and $\left\{y_{n}\right\}$ are diverging.

Let us find remarks of this sequence. Let us use following equation:

$$
\ln (n)=2.30259 \times \lg n, n=1,2,3, \ldots
$$

The most common method of evaluating natural logarithms is by a scientific notation calculation. Use this calculator to check following values:

$$
\ln (2)=0.69315 \ldots, 1+\frac{1}{2}=1.5
$$

Consequently,

$$
\begin{gathered}
\ln 3=1.09861,1+\frac{1}{2}+\frac{1}{3}=1 \frac{5}{6}, \\
\ln 4=1.38630,1+\frac{1}{2}+\frac{1}{3}+\frac{1}{4}=2 \frac{1}{12}, \\
\ln 5=1.60944,1+\frac{1}{2}+\frac{1}{3}+\frac{1}{4}+\frac{1}{5}=2 \frac{17}{60},
\end{gathered}
$$

$$
\ln 6=1.79176,1+\frac{1}{2}+\frac{1}{3}+\frac{1}{4}+\frac{1}{5}+\frac{1}{6}=2 \frac{9}{20}
$$$$
\ln 7=1.94591,1+\frac{1}{2}+\frac{1}{3}+\frac{1}{4}+\cdots+\frac{1}{7}=2 \frac{83}{140} \text {, }
$$$$
\ln 8=2.07945,1+\frac{1}{2}+\frac{1}{3}+\frac{1}{4}+\cdots+\frac{1}{8}=2 \frac{201}{280}
$$$$
\ln 9=2.19723,1+\frac{1}{2}+\frac{1}{3}+\frac{1}{4}+\cdots+\frac{1}{9}=2 \frac{2089}{2520}
$$

$\ln 10=2.303259,1+\frac{1}{2}+\frac{1}{3}+\frac{1}{4}+\cdots+\frac{1}{10}=2 \frac{2341}{2520}$, 
$\ln 11=2.398,1+\frac{1}{2}+\frac{1}{3}+\frac{1}{4}+\cdots+\frac{1}{11}=3 \frac{551}{27720}$

$\ln 15=2.70806,1+\frac{1}{2}+\frac{1}{3}+\frac{1}{4}+\cdots+\frac{1}{15}=3 \frac{114677}{360360}$

$\ln 20=2.99574,1+\frac{1}{2}+\frac{1}{3}+\frac{1}{4}+\cdots+\frac{1}{20}=3.59774$,

$\ln 30=3.401205,1+\frac{1}{2}+\frac{1}{3}+\frac{1}{4}+\cdots+\frac{1}{30}=3.994987$,

$\ln 40=3.68889,1+\frac{1}{2}+\frac{1}{3}+\frac{1}{4}+\cdots+\frac{1}{40}=4.278542$,

$\ln 50=3.912031,1+\frac{1}{2}+\frac{1}{3}+\frac{1}{4}+\cdots+\frac{1}{50}=4.499205$,

$\ln 60=4.094353,1+\frac{1}{2}+\frac{1}{3}+\frac{1}{4}+\cdots+\frac{1}{60}=4.679870$,

$\ln 70=4.248504,1+\frac{1}{2}+\frac{1}{3}+\frac{1}{4}+\cdots+\frac{1}{70}=4.832837$,

$\ln 80=4.382036,1+\frac{1}{2}+\frac{1}{3}+\frac{1}{4}+\cdots+\frac{1}{80}=4.965659$,

$\ln 90=4.499819,1+\frac{1}{2}+\frac{1}{3}+\frac{1}{4}+\cdots+\frac{1}{90}=5.082751$,

$\ln 100=4.60518,1+\frac{1}{2}+\frac{1}{3}+\frac{1}{4}+\cdots+\frac{1}{100}=5.104609$,

Then we hold following

$$
\begin{gathered}
z_{1}=1-\ln 1=1, \\
z_{2}=1+\frac{1}{2}-\ln 2=0.80685, \\
z_{3}=1+\frac{1}{2}+\frac{1}{3}-\ln 3=0.7347233, \\
z_{4}=1+\frac{1}{2}+\frac{1}{3}+\frac{1}{4}-\ln 4=0.69703, \\
z_{5}=1+\frac{1}{2}+\frac{1}{3}+\frac{1}{4}+\frac{1}{5}-\ln 5=0.673893, \\
z_{6}=1+\frac{1}{2}+\frac{1}{3}+\frac{1}{4}+\frac{1}{5}+\frac{1}{6}-\ln 6=0.65824, \\
z_{7}=1+\frac{1}{2}+\frac{1}{3}+\frac{1}{4}+\frac{1}{5}+\frac{1}{6}+\frac{1}{7}-\ln 7=0.64695, \\
z_{8}=2.71786-2.07945=0.63841, \\
z_{9}=2.82897-2.19723=0.63174, \\
z_{10}=2.92897-2.30259=0.62638, \\
\ldots \ldots \ldots \ldots \ldots \ldots \ldots \ldots \ldots \ldots \ldots \ldots \ldots \ldots \ldots \\
z_{15}= \\
z_{20}=3.318229-2.70806=0.61017,
\end{gathered}
$$

$$
\begin{aligned}
& z_{30}=3.99499-3.401205=0.593783 \\
& z_{100}=5.104609-4.61518=0.489429
\end{aligned}
$$

That is, we have verified that, the domain of the definition of this function is $[1,+\infty)$ and the value of this function is $(0,1]$. As the concept of function is of paramount importance in mathematics and among other disciplines as well, we would like to extend our introduction about function from where we finished studying sequence. Note that, when one must find limit for different function, then one must be careful to study it. One of the key steps in determining this limit is the ability to sketch their graphs. This step involved in sketching the graph of a function is to consider the behavior of the functions for large values of $x$. Hence if we look given sequences $\mathrm{x} \_\mathrm{n}$ and $\mathrm{y} \_\mathrm{n}$ as functions, then these functions are approaching, but they are not intersecting. One of important feature of these functions is that they are strictly monotonic- that is they are always increasing. Moreover I can use this behaviour in terms of limits. $[4,6,12]$

In the discussion that follows we will consider an alternative approach to the notion of a limit that is based on analytical concepts rather than on intuitive concepts. Now let us use following partial sums by Euler transformation

$1,0.19315,0.07213,0.02314,0.01565,0.01129, \ldots$

$0.80685,0.12102,0.04899,0.00749,0.00436, \ldots$

$0.68583,0.07203,0.0415,0.00313, \ldots$

One can easy see that, these series tend to zero. [7-9]

L'Hopital's rule will enable us to determine such limits when the differential coefficients of the denominator and the numerator can be found. Then we hold following

$$
\begin{aligned}
& \lim _{n \rightarrow \infty}\left(\sum_{k=1}^{n} \frac{1}{k}-\ln n\right)= \\
& =\lim _{n \rightarrow \infty}\left(\ln e^{\left(\sum_{k=1}^{n} \frac{1}{k}\right)}-\ln n\right)= \\
& =\lim _{n \rightarrow \infty}\left(\ln \frac{e^{\left(\sum_{k=1}^{n} \frac{1}{k}\right)}}{n}\right)= \\
& =\lim _{n \rightarrow \infty}\left(\ln \frac{e^{\left(\sum_{k=1}^{n} \frac{1}{k}\right)}}{n}\right)= \\
& =\lim _{n \rightarrow \infty}\left(\frac{n}{e^{\left(\sum_{k=1 k}^{n}\right)}}\right) \times\left(\frac{n \times\left(e^{\left(\sum_{k=1}^{n}\right)}\right)-e^{\left(\sum_{k=1}^{n} \frac{1}{k}\right)}}{n^{2}}\right)= \\
& =\lim _{n \rightarrow \infty}\left(\frac{n}{e^{\left(\sum_{k=1 \frac{1}{k}}^{n}\right)}} \times \frac{-e^{\left(\sum_{k=1}^{n} \frac{1}{k}\right)}}{n^{2}}\right)= \\
& \lim _{n \rightarrow \infty}\left(-\frac{1}{n}\right)=\lim _{n \rightarrow \infty}\left(\frac{1}{n^{2}}\right)=\lim _{n \rightarrow \infty}\left(-\frac{2}{n^{3}}\right)=\cdots=0 \text {. }
\end{aligned}
$$

Being $e^{\left(\sum_{k=1}^{n} \frac{1}{k}\right)}$ is any real number, then it is clear that in 
this way one can find the derivatives of any order by successively applying the its formula. Thus its all derivatives are equal to zero. $[8-11,15]$

\section{Conclusion}

Analysis is used extensively in mathematics, as well as in real live. I must note that, definite integration which involves integration by parts requires attention to detail in notation about use of the limit. The purpose of this paper, like the majority of mathematics conception, consists of ordinary purpose and a number of special views from the theories being discussed. Along with the special views, which will be introduced as needed, we use common properties of the limit conception. One knew that, the limit conception means that, one must obtain expression for "sequence of approximate values". In this edition the paper has been devoted in the following five convenient categories: sequence, natural logarithms, graphs of the function, Euler transformations and l'Hopital's rule. We continue our introduction of the theory of the limits by considering the limit of a function of a natural-number argument (a sequence), in view of the fundamental role played by this limit, as already explained, and also because all the basic facts of the theory of limits can actually be clearly seen in this given situation.

\section{Acknowledgements}

Rena E. Kerbalayeva acknowledgement the support of Baku State University and as well as National Academy Science of Azerbaijan. The author is grateful to the referees for numerous comments that improved the quality of the paper.

\section{References}

[1] Alan S. Tussy., R. David Gustatson., Diane R. Koing.: Basic Mathematics for college students, fourth education, (2011), pp. 594-637.
[2] Elias Zakon.: Mathematical Analysis, vol. 1, (2011), pp. 149314.

[3] Fabio Cirrito., Nigel Buckle., Iain Dunbar.: Mathematics Higher Level, (2007).

[4] Hutchinson John E.: Introduction To Mathematics Analysis, Department of Mathematics School of Mathematical Scences ANU, 6/7 (1995), pp. 38-41, 77-87.

[5] Gowers T.: the Princeton Companion to Mathematics (Princeton University Press, 2008).

[6] Jean Linsky., James Nicholson., Brian Western.: Complete Pure Mathematics 213 for Campridge International AS\&Level, (2018).

[7] Jonathan Wicket., Kemper Lewis.: An introduction to Merchanical Engineering, third education, (2013).

[8] Qiu-Ming Luo.: Journal of Integer Sequences, vol., 12, (2009), pp. 1-8.

[9] Ralph Palmer Agnew.: Euler Transformation, American Journal of Mathematics, v. 66, №2, (1944), pp. 313-338.

[10] Thomas J. Osler.: Partial sums of series that cannot be an integer, The Mathematic Gazette 96, November, (2012), pp. 515-519,

[11] Thomas Schmelzer., Robert Baillie.: Summing a Curious, Slowly Convergent Series, The American Mathematical Montly, 115, 6, (2008), pp. 540-545.

[12] Tony Beadsworth.: Complete Additional Mathematics for Campridge IGCSE\&0level, (2017), pp. 320-332, 151-224.

[13] Vladimir A. Zorich.: Mathematical Analysis I. Springer (2002), pp. 79-147.

[14] Weisstein E. W.: CRC Concise Encyclopedia of Mathematics; English Edution; $2^{\text {nd }}$ Eduation (CRC Press, Kindle version, 1998).

[15] William F. Trench.: Introduction to real Analysis, (2013), pp. 30-53, 88-98, 179-281. 